\title{
Actores de la cadena productiva que deben participar para prevenir el uso de clembuterol en la engorda del ganado bovino en México
}

Productive chain actors who should participate to prevent the use of clembuterol in bovine cattle feeding in México

Delgado Prado Emma Daniela ${ }^{1}$, Córdova-Izquierdo Alejandro ${ }^{2 凶}$, García Díaz Miguel Ángel ${ }^{3}$, Espinosa Cervantes Román ${ }^{2}$, Ruiz Lang Claudio Gustavo ${ }^{2}$, Iglesias Reyes Adrian Emmanuel ${ }^{4}$, Villa Mancera Edmundo Abel $^{5}$, Huerta Crispín Rubén ${ }^{5}$, y Rodríguez Denis Blanca Estela ${ }^{1}$

${ }^{1}$ Práctica privada. ${ }^{2}$ Departamento de Producción Agrícola y Animal. Universidad Autónoma Metropolitana Unidad Xochimilco, Ciudad de México. ${ }^{3}$ SENASICSAGARPA.MÉXICO. ${ }^{4}$ Maestría en Ciencias Agropecuarias. UAM-Xochimilco, Ciudad de México. ${ }^{5}$ Facultad de Veterinaria. Benemérita Universidad Autónoma de Puebla, México.

${ }^{凶}$ Autor para correspondencia: acordova@correo.xoc.uam.mx

Recibido: $18 / 02 / 2017$

Aceptado: 30/06/2017

\section{RESUMEN}

El clembuterol, Químicamente se describe como polvo blanco, anhidro, muy soluble en agua y altamente estable a temperatura ambiente, su punto de fusión es de 174 a $175.5^{\circ} \mathrm{C}$. También es un derivado sintético perteneciente a una clase de medicamentos análogos fisiológicamente a la adrenalina, tiene la capacidad de interactuar con receptores adrenérgicos, generalmente del tipo $\beta_{2}$ ( $\beta$ agonista), es uno de los modificadores metabólicos más conocido en el área de producción de carne, debido al alto grado residual que deja esta sustancia en los tejidos comestibles y sus posibles repercusiones en la salud pública. En este trabajo, se describen los principales actores de la cadena productiva en la engorda de ganado que deben participar para prevenir el uso de clembuterol. La información fue recopilada de información científicas y publicaciones de las autoridades competentes, tales como: SENASICA, SAGARPA, COFEPRIS y Ley Federal de Sanidad Animal.

Palabras clave: Ganado bovino, engorda, clembuterol.

\begin{abstract}
Clenbuterol, chemically described as a white, anhydrous powder, very soluble in water and highly stable at room temperature, has a melting point of 174 to $175.5^{\circ} \mathrm{C}$. It is also a synthetic derivative belonging to a class of drugs physiologically analogous to adrenaline, has the ability to interact with adrenergic receptors, generally of the $\beta 2$ (agonist) type, is
\end{abstract}


one of the metabolic modifiers best known in the area of meat production, Due to the high residual degree that this substance leaves in the edible tissues and its possible repercussions in the public health. In this paper, we describe the main actors of the productive chain in cattle fattening that must participate to prevent the use of clenbuterol. The information was compiled from scientific information and publications of the competent authorities, such as: SENASICA, SAGARPA, COFEPRIS and Federal Law of Animal Health.

Keywords: Bovine cattle, fattening, clenbuterol.

\section{INTRODUCCIÓN}

En los Estados Unidos de América, el uso del clembuterol en la alimentación para la engorda del ganado bovino está prohibido. Por otro lado, la Unión Europea prohíbe el uso de sustancias hormonales $\mathrm{y}$ beta-agonistas con fines promotores del crecimiento (Jiménez et al., 2011). Para incrementar la producción pecuaria se recurre en forma constante a la utilización de moduladores del crecimiento, dichas sustancias tales como prebióticos, hormonas, antimicrobianos, modificadores metabólicos, entre otros, mejoran en primer lugar parámetros productivos y culminan en mayor cantidad de productos (Gómez, 2014). En la producción animal se debe considerar a la seguridad alimentaria, pero a la vez procurar que los alimentos agropecuarios sean inocuos, que garanticen que no dañan la salud de los consumidores (Valladares et al., 2015).

Las cadenas productivas o agroalimentarias se han utilizado para la elaboración de diagnósticos integrales de un producto agrícola, ganadero y/o acuícola (Cuevas, 2011). Estas son constituidas por actores interrelacionados a través de una sucesión de operaciones, de producción, transformación y comercialización. Los principales actores que participan en estas cadenas productivas son: en primer lugar los comerciantes quienes venden los becerros a los productores; en segundo lugar están los productores, estos se encargan de la engorda del ganado; el tercer actor son los proveedores de diferentes insumos: ya sea de semillas, alimento, medicamento, entre otros; el cuarto actor de la cadena

productiva corresponde al de procesadores, quienes se encargan de la compra de toros y vacas de desecho para después hacer la transformación de los animales en pie a carne en canal; el quinto actor son los mayoristas (almacenistas) y los minoristas estas pueden ser las carnicerías (comerciante) quienes se encargan de vender cortes de carne, embutidos al consumidor o usuario final; finalmente se encuentra el consumidor, quien compra el producto en los diferentes puntos de venta para su propio consumo (Calderón et al., 2012), no obstante, los consumidores exigen productos cárnicos de mejor calidad en cuanto a color, jugosidad y menor deposición de grasa, lo que en gran parte ha obligado al productor a utilizar el

clembuterol para obtener estas características $(\mathrm{Ku}, 2011)$. En la cadena productiva existen eslabones que se encargan de aportar y ayudar a los actores, brindándoles planes, programas y leyes que pueden impactar positiva y/o negativamente el desempeño de su producción (Calderón et al., 2012). Estos actores deben participar en le prevención de clembuterol, ya que es uno de los 
modificadores metabólicos más conocido en el área de producción de carne, debido al alto grado residual que deja esta sustancia en los tejidos y sus posibles repercusiones en la salud pública (Domínguez-Vara et al., 2009). En México, se han emitido normas que prohíben el empleo del clembuterol en la alimentación animal (bovinos, cerdos, ovinos y aves), su importación, comercialización, transporte y suministro. $\mathrm{Su}$ uso se continúa dando de manera clandestina en animales de engorde destinados al consumo humano, por eso es necesario revalorar los peligros potenciales que puede ocasionar al emplearse tanto en medicina humana como en veterinaria (Ley Federal de Sanidad Animal, 2007).

\section{DESARROLLO}

El clembuterol está prohibido por la ley para su uso en la alimentación de ganado bovino. Su presencia en los productos y subproductos cárnicos representa un riesgo en la salud del consumidor ya que, al usarlo como promotor de crecimiento en la engorda de ganado bovino, es causa de intoxicación en la población que consume carne y vísceras, principalmente el hígado (SENASICA, 2015).

Químicamente se describe como polvo blanco, anhidro, muy soluble en agua y altamente estable a temperatura ambiente, su punto de fusión es de 174 a $175.5^{\circ} \mathrm{C}$. También es un derivado sintético

perteneciente a una clase de medicamentos análogos fisiológicamente a la adrenalina, tiene la capacidad de interactuar con receptores adrenérgicos, generalmente del tipo $\beta 2$ ( $\beta$ agonista) (Valladares et al., 2014).
El término cadena productiva o agroalimentaria a menudo se utiliza para sustituir otros conceptos utilizados en el mundo de los negocios para mejorar la competitividad, como el de "cadena de valor", "cadena de suministro", y "sistema producto". Sin embargo, el enfoque de cadena agroalimentaria se ha utilizado para la elaboración de diagnósticos integrales de un producto agrícola, ganadero y/o acuícola. En el caso de un sistema de producción (agrícola, pecuario o forestal) involucra actores, factores y acciones que se ubican antes, dentro y después de la unidad de producción primaria. Antes de ésta se hallan los proveedores de los diferentes insumos: semillas, máquinas, fertilizantes, plaguicidas, etc. Dentro de las explotaciones primarias se localizan los diferentes tipos de productores. Después de las fincas están los procesadores, le siguen los mayoristas y minoristas y al final están los consumidores. El conjunto de estos diferentes grupos de actores y sus actividades, constituye lo que se llama cadena productiva, que puede ser una cadena agroalimentaria (Cuevas, 2011).

Las cadenas productivas o agroalimentarias se han utilizado para la elaboración de diagnósticos integrales de un producto agrícola, ganadero y/o acuícola (Cuevas, 2011). Estas son constituidas por actores interrelacionados a través de una sucesión de operaciones, de producción, transformación y comercialización.

Los principales actores que participan en estas cadenas productivas son:

Comerciantes, son quienes venden los becerros a los productores, pero esta venta se realiza de manera independiente, o considerando sólo los Intereses del productor, también los comerciantes 
realizan en forma tradicional, ofertando el producto en pie, ya sea en el rancho o en algún mercado regional (Hernández et al., 2011).

Productores, estos se encargan de la engorda del ganado, generalmente los becerros con pesos entre 160-180 kg

continúan su crecimiento bajo condiciones de pastoreo con o sin suplementación alimenticia hasta alcanzar los 250-300 kg., momento en que son enviados a los corrales de engorda. Es importante destacar que el resultado final en el comportamiento de los bovinos en corral, dependerá de varios factores, entre los que destacan: Alimentación (Tipo de dietas e ingredientes usados), el uso de anabólicos y beta-agonistas legalmente permitidos, edad, sexo y genotipo de los

animales, condiciones ambientales, manejo a la recepción y durante el periodo de engorda, días de finalización, e Instalaciones (Aguilera, 2010).

Proveedores de diferentes insumos, son aquellos que venden, ya sea semillas, alimento para la engorda del ganado, maquinarias para la Unidad de Producción, medicamento para los bovinos (Calderón et al., 2012).

Procesadores, quienes se encargan de la compra de toros y vacas de desecho para después hacer el sacrificio o la transformación de los animales en pie a carne en canal. El sacrificio se realiza en el rastro o matadero municipal; éste se considera como un agente indirecto, ya que sólo proporciona los servicios de sacrificio y maquila, pero no interviene en los precios del mercado (Vilaboa et al., 2009). Normalmente, la carne que sale de un rastro TIF es más higiénica por contar con inspecciones veterinarias, por el mayor número de controles sanitarios, y porque se mantiene en refrigeración, ya que generalmente tiene como objetivo final la venta en cadenas de supermercados, carnicerías selectas o exportación. La carne de rastro municipal está destinada a carnicerías locales y a mercados sobre ruedas, donde en la mayoría de los casos no se mantiene en refrigeración (SAGARPA, 2012).

Mayoristas (almacenistas o tiendas de autoservicio), éstos comercializan $46 \%$ de la venta de alimentos en México. Se cuenta con grandes cadenas de autoservicio como: Wal-Mart, Soriana, Chedraui y Comercial Mexicana; clubes como Costco y Sam's Club. El

establecimiento de grandes supermercados regionales ha facilitado el rápido crecimiento en las importaciones de carne, debido a la facilidad del manejo, a la infraestructura de transporte y almacenamiento, así como a su bajo precio (Olaya, 2012). En los últimos años se ha observado en México una expansión en la participación de las tiendas de autoservicio en el comercio al menudeo (Castañeda, 2011).

Minoristas, éstos pueden ser las carnicerías quienes se encargan de vender cortes de carne, embutidos, al consumidor o usuario final (Calderón et al., 2012). Este centro de comercialización de la carne ha aumentado como consecuencia del crecimiento de las ciudades y de las áreas urbanas, en cuyas carnicerías se ofrece carne fresca y en algunas de ellas se procesan algunos tipos de carne. $\mathrm{Su}$ importancia radica en que se encuentran en prácticamente en todas las colonias y áreas urbanas de las ciudades (Olaya 2012). Las carnicerías venden $71 \%$ carne de res y de cerdo, mientras que dentro del $29 \%$ restante, el $68 \%$ vende exclusivamente carne de res, y solo el 
$32 \%$ únicamente carne de cerdo (INIFAP 2015).

Consumidor, quien compra el producto en los diferentes puntos de venta para su propio consumo (Calderón et al., 2012) no obstante, los consumidores exigen productos cárnicos de mejor calidad en cuanto a color, jugosidad y menor deposición de grasa, lo que en gran parte ha obligado al productor a utilizar el

clembuterol para obtener estas características (Ku, 2011).

En la cadena productiva existen eslabones que se encargan de aportar y ayudar a los actores, brindándoles planes, programas y leyes que pueden impactar positiva $\mathrm{y} / \mathrm{o}$ negativamente el desempeño de su producción (Calderón et al., 2012). En la actualidad hay algunos productores que con las técnicas convencionales de alimentación no logran el peso que desean en el ganado, por lo que se ven motivados a usar en la alimentación sustancias promotoras de crecimiento prohibidas por la Ley Federal de Sanidad Animal y su Reglamento, estas sustancias representan un riesgo en la salud del consumidor, por lo que la Secretaría de Agricultura, Ganadería, Desarrollo Rural,
Pesca y Alimentación (SAGARPA) a través del Servicio Nacional de Sanidad, Inocuidad y Calidad Agroalimentaria (SENASICA) en coordinación con las Delegaciones de la SAGARPA en los Estados, se creó el programa preventivo en la producción de carne bovina, llamado Proveedor Confiable (Libre de Clembuterol), cuyo propósito es brindar la garantía a los consumidores que todo producto cárnico salido de un corral de engorda, estará libre de clembuterol (SENASICA, 2015).

También la Secretaría de Salud (SSA) lleva a cabo actividades referentes a la inocuidad de los productos cárnicos a través de la Comisión Federal para la Protección Contra Riesgos Sanitarios (COFEPRIS), y por la Secretaría de Agricultura, Ganadería, Desarrollo Rural, Pesca y Alimentación (SAGARPA), a través del Servicio Nacional de Sanidad, Inocuidad y Calidad Agroalimentaria (SENASICA), de acuerdo con sus ámbitos de competencia, en el cumplimiento respectivo de las leyes, reglamentos y normas oficiales mexicanas en dicha materia (Jiménez et al., 2011). 
Delgado et al., 2017

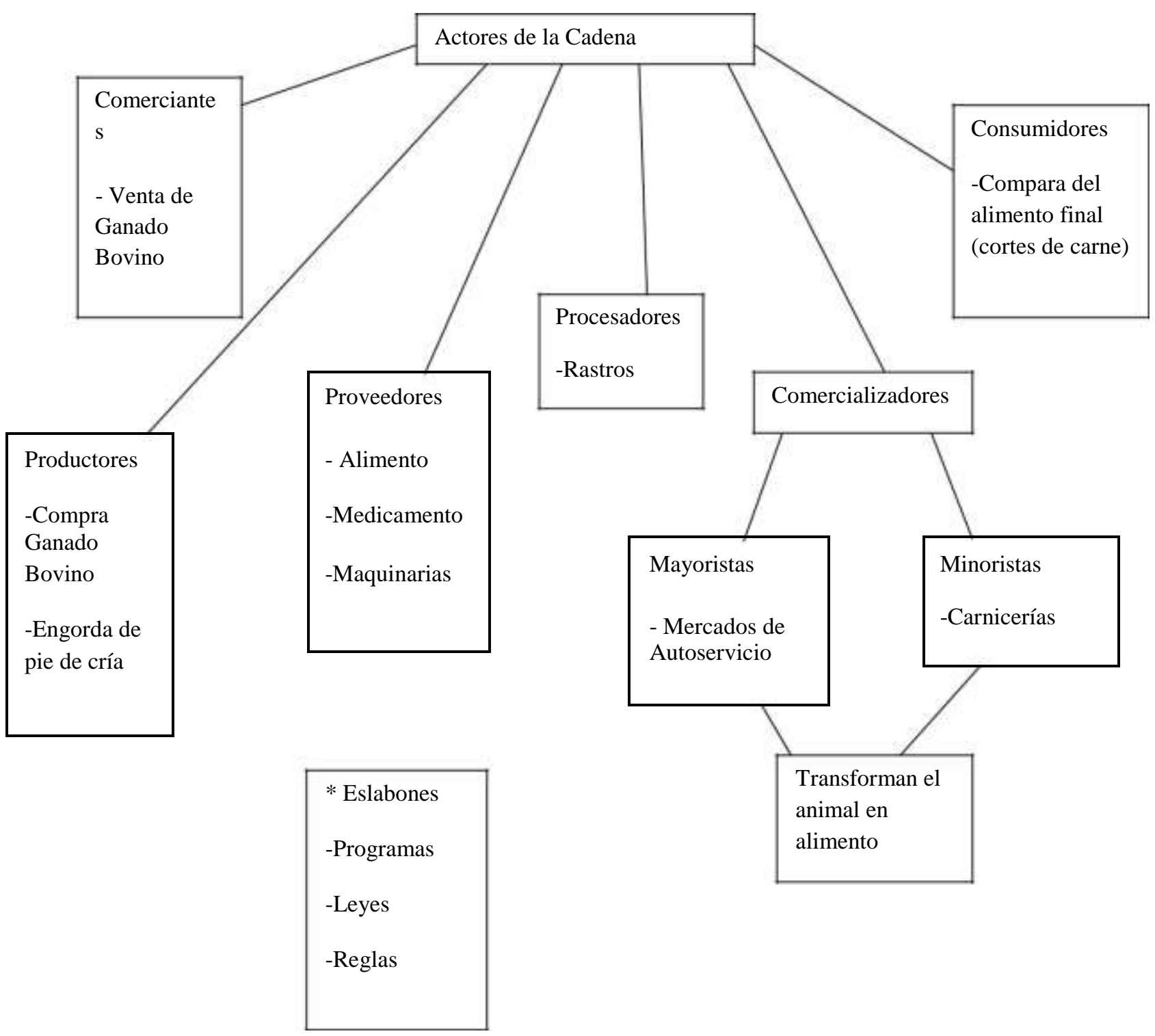




\section{CONCLUSIONES}

Los productores deben de tener conocimiento de las Buenas Prácticas Pecuarias e implementarlas durante el proceso de engorda del ganado bovino, ya que las Buenas Prácticas Pecuarias son usadas como una medida de control para prevenir problemas de salud y calidad de los alimentos, constituyen la base para establecer cualquier programa de aseguramiento de la inocuidad.

En general se puede decir que los productores, pueden evitar el uso de clembuterol, ya que pueden inscribirse al Programa de Proveedor Confiable y tener conocimiento de las Buenas Prácticas

Pecuarias, y así brindarles el conocimiento de que el uso de clembuterol no genera una importante ganancia económica. En la actualidad, los productores pueden tener el registro de productor confiable por parte SENASICA de la SAGARPA, ya que permite el registro de unidades de producción pecuaria que cuenten con la certificación de hato libre de residuos de clembuterol ayuden en la mejora de la salud pública y exista un alimento inocuo.

En conclusión, se puede decir que los diferentes actores pueden participar en la prevención del uso de clembuterol para la alimentación del ganado en engorda, los productores tienen que tener una amplia información sobre el alimento que se les proporciona al ganado y sobre las Buenas Prácticas Pecuarias que la SAGARPA recomienda, los proveedores tienen que certificar que el alimento que vendan no contenga clembuterol, los procesadores (rastros) deben ser TIF para que los comercializadores puedan vender la carne inocua y libre de clembuterol a los consumidores para su satisfacción. En cuanto al Programa de Proveedor Confiable se espera que todas las unidades de producción primaria (engorde de ganado bovino) deben estar inscritas en este Programa.

\section{RECOMENDACIONES}

Que los eslabones que se encargan de aportar y ayudar a los actores, brindándoles planes, programas y leyes, cumplan con estos y se lleven a cabo correctamente.

Los minoristas (carnicerías) compren con proveedores que estén dentro del Programa de Proveedor Confiable, para garantizar un producto inocuo.

Que los actores conozcan de la Buenas Prácticas Pecuarias, para asegurar la calidad e inocuidad de los alimentos.

Que los comerciantes del sector cárnico tengan mayor información sobre el proceso de venta y los pasos que sigue el consumidor para comprar un producto con el fin de prestar un mejor servicio a sus clientes.

\section{LITERATURA CITADA}

Aguilera S.R., 2010. Factores críticos para hacer rentable la finalización de bovinos. IV Congreso Rentabilidad de la Ganadería de Carne. Veracruz. Revista Veracruz Pecuario, pp. 2-6.

Calderón J. et al. 2012. Estructura y función de la cadena productiva de carne de bovino en la ganadería ejidal de Tecpatán, Chiapas, México. Revista Avances en Investigación Agropecuaria 16(2): 45-61 
Castañeda S. A. 2011. Supermercados. Competencia en precios. Economía Mexicana Nueva Época, vol. XXI, núm. 2, pp. 297-349

Cuevas, R.V. 2011. Análisis del enfoque de cadenas productivas en México.

Universidad Autónoma Chapingo. Revista Chapingo Serie Horticultura No. 56, pp.83-94.

Domínguez-Vara, I. A., J. MondragónAncelmo, M. González R., F. SalazarGarcía, J. L. Bórquez-Gastelum, y A. Aragón-Martínez. 2009. Los $\beta$-agonistas adrenérgicos como modificadores metabólicos y su efecto en la producción, calidad e inocuidad de la carne de bovinos y ovinos: una revisión. Ciencia Ergo Sum 16 (3) 278-284.

Gómez P. E., 2014. La biotecnología aplicada a la producción animal en las Américas. Cultura, Ciencia y Tecnología. ASDOPEN-UNMSM, No. 6.

Hernández M. J., Rebollar R. S., González R. F., Guzmán S. E., Albarrán P. B., García M.A. 2011. La cadena productiva de ganado bovino en el Sur del Estado de México. Revista Mexicana de Agronegocios, vol. XV, núm. 29, pp. 672-680.

INIFAP. 2015. Calidad en puntos de venta de Carne. Folleto Técnico No. 22

Jiménez S.L., Garza R. J., Sumano L. H., Fragoso S., H. 2011. Vigilancia sanitaria en el uso ilícito del clembuterol y su coordinación intersectorial en dos entidades de México. Vet. Méx., 42 (1).

$\mathrm{Ku}$ Vera J., 2011. Clenbuterol: su uso en medicina veterinaria y producción animal. Cuerpo Académico Producción Animal en Agroecosistemas Tropicales. Departamento de Nutrición Animal. Campus de Ciencias Biológicas y Agropecuarias - UADY. Vol. 4 No. 1

Ley Federal de Sanidad Animal. México. 2007 (Diario Oficial de la Federación 257-2007. Ultima modificación 2012).

Olaya F. E. 2012. Residuos de clembuterol en Tejidos Comestibles de Bovino. Chapingo, Estado de México. Tesis.

SAGARPA. 2012. Carne de Res Mexicana. Folleto Técnico No. 15

SENASICA. 2015. Programa de proveedor confiable (libre de clembuterol). Guía de operación.

Valladares C.B., Bañuelos V.R., Peña B.S.D., Velázquez Ordoñez V, Echavarría-Cháirez FG, Muro-Reyes A, Ortega-Santana C. 2015. Riesgos a la salud por el uso de clorhidrato de clembuterol: una revisión. Rev Med Vet. (30): 139-49

https://doi.org/10.19052/mv.3618

Valladares C.B., Bañuelos V.R., Peña, B.S.D., Velázquez, O.V., Velázquez, A.Y. y Nava, O.A. 2014. Illegal use of clenbuterol in cattle production in México. Health, 6:673-676.

https://doi.org/10.4236/health.2014.68087

Vilaboa A. J., Díaz R. P., Platas R. D., Ruiz R. O., González M. S., Juárez L. F. 2009. Estructura de comercialización de bovinos destinados al abasto de carne en la región del Papaloapan, Veracruz, México. Economía, Sociedad y Territorio, vol. Economía, Sociedad y Territorio Vol. IX, núm. 31, 831-854. 
Copyright (c) 2017 Emma Daniela Delgado Prado, Alejandro Cór dova Izquierdo, Miguel Ángel Garcia Diaz, Rom án Espinosa Cervantes, Claudio Gustavo Ruiz Lang, Adrián Emmanuel Iglesias Reyes, Edm undo Abel Villa Mancera,

Rubén Huerta Crispin y Blanca Estela Rodriguez Denis

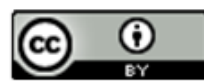

Este tex to está protegido por una licencia licencia Creative Commons 4.0.

Usted es libre para Compartir —copiar y redistribuir el material en cualquier medio o form ato- y Adaptar el documento —remezclar, transformar y crear a partir del material- para cualquier propósito,, incluso para fines comerciales, siempre que cumpla la condición de:

Atribución: Usted debe dar crédito a la obra original de manera adecuada, proporcionar un enlace a la licencia, e indicar si se han realizado cam bios. Puede hacerlo en cualquier form a razonable, pero no de form a tal que sugiera que tiene el apoyo del licenciante o 10 recibe por el uso que hace de la obra.

$\underline{\text { Resumendelicencia }}$ - Textocompletodelalicencia 